\title{
Recurrent Syncope due to Esophageal Squamous Cell Carcinoma
}

\author{
A. Casini ${ }^{\mathrm{a}}$ E. Tschanz ${ }^{\mathrm{b}}$ P.Y. Dietrich ${ }^{\mathrm{c}} \quad$ M. Nendaz ${ }^{\mathrm{a}}$ \\ Departments of a Internal Medicine, ${ }^{\mathrm{b}}$ Pathology, and 'Oncology, Geneva University \\ Hospitals, Geneva, Switzerland
}

\section{Key Words}

Aortic stenosis · Syncope $\cdot$ Carotid sinus syndrome $\cdot$ Esophageal carcinoma

\begin{abstract}
Syncope is caused by a wide variety of disorders. Recurrent syncope as a complication of malignancy is uncommon and may be difficult to diagnose and to treat. Primary neck carcinoma or metastases spreading in parapharyngeal and carotid spaces can involve the internal carotid artery and cause neurally mediated syncope with a clinical presentation like carotid sinus syndrome. We report the case of a 76-year-old man who suffered from recurrent syncope due to invasion of the right carotid sinus by metastases of a carcinoma of the esophagus, successfully treated by radiotherapy. In such cases, surgery, chemotherapy or radiotherapy can be performed. Because syncope may be an early sign of neck or cervical cancer, the diagnostic approach of syncope in patients with a past history of cancer should include the possibility of neck tumor recurrence or metastasis and an oncologic workout should be considered.
\end{abstract}

\section{Introduction}

Syncope is defined as a transient loss of consciousness that occurs spontaneously and is rapidly reversible with minimal intervention. It is caused by a wide variety of disorders that range from common benign disorders to severe life-threatening diseases. Even when an extensive evaluation of syncope is undertaken, its cause may be difficult to diagnose; data from Sarasin et al. [1] show unexplained syncope in $20 \%$ or more of patients with syncope in the emergency department despite standardized evaluation. Carotid sinus syndrome, a variety of neurally mediated syncope, is rarely associated with malignancy. We describe a patient who suffered from recurrent syncope due to tumor compression of the carotid sinus by metastases of a carcinoma of the esophagus, successfully treated with radiotherapy. 


\section{Case Report}

A 76-year-old man was admitted to our hospital for recurrent syncope occurring while sitting or standing, preceded by headache and followed by circumstantial amnesia without neurological deficits. Two years before the admission, he was treated with chemotherapy and radiotherapy for a squamous cell carcinoma of the middle third of the esophagus with mediastinal lymph node metastasis; after 16 months, he received second-line chemotherapy because of tumor recurrence. On admission, temperature was $36.5^{\circ} \mathrm{C}$, pulse 90 beats $/ \mathrm{min}$, and blood pressure $134 / 78 \mathrm{~mm} \mathrm{Hg}$. He did not report any chest pain, exertional dyspnea or palpitation. He did not take any antihypertensive drugs. The laboratory tests and chest X-ray were unremarkable; the EKG showed an atrial fibrillation and signs of ventricular hypertrophy without ischemia. During hospitalization, the patient had many syncope recurrences. One episode, characterized by loss of consciousness for $30 \mathrm{~s}$ associated with a gaze deviation to the left, diaphoresis, pallor, and hypotension $(60 / 30 \mathrm{~mm} \mathrm{Hg})$ without bradycardia, occurred during a 24-hour electrocardiographic monitoring. The latter was normal, thus excluding arrhythmia as a cause of this episode. A transthoracic echocardiography showed a low-gradient aortic stenosis (valve area $1 \mathrm{~cm}^{2}$, mean gradient $16 \mathrm{~mm} \mathrm{Hg}$, aortic jet velocity $3.1 \mathrm{~m} / \mathrm{s}$ ) and normal ejection fraction of the left ventricle with important concentric hypertrophy; an exercise SPECT study with thallium and cardiac MRI excluded myocardial ischemia. As symptoms were not typical for valvular abnormality as a single etiology and the patient had a past history of ischemic stroke, neurological investigations were completed by EEG, brain MRI, and carotid-vertebral duplex Doppler sonography: all these investigations yielded normal results. Finally, an upright tilt-table test was performed, showing a fall in the arterial blood pressure reproducing symptoms without any change in heart rate, consistent with reflex syncope of vasodepressor type (ig. 1). The massage of carotid sinus did not induce any symptoms. A cervical computed tomography (CT) showed the presence of a cervical mass infiltrating the sheath of the right common carotid artery just below and in contact with the carotid bifurcation, without involvement of the retropharyngeal space. We renounced to any histological samples of this mass because an upper gastrointestinal endoscopy confirmed a relapse of the esophageal cancer (fig. 2), proven by histopathologic examination of a biopsied specimen (fig. 3 ). Given the poor condition of the patient, we opted for a targeted radiotherapy treatment on the right carotid sheath. Once this treatment began, he did not present any recurrence of syncope. He finally received 5 sessions (30 Gy in total) and eventually died 6 weeks later of an episode of severe broncho-aspiration.

\section{Discussion}

The initial evaluation of a patient presenting with syncope consists of careful history, physical and electrocardiographic examination. Multiple potential causes are a common situation in the elderly population. Our patient presented many clinical features that initially suggested a cardiovascular syncope, like abnormal EKG and presence of definite valvular heart disease. However, because of an atypical presentation for a syncope due to valvular disease, we continued the investigations, according to the recently published ESC guidelines for the diagnosis and management of syncope [2]. We performed an upright tilt-table test which turned out positive and led us to look for a malignant etiology in the context of this patient.

Neurally mediated syncope (also named reflex syncope) is the most common group of disorders causing syncope. These disorders result from reflex-mediated modifications in vascular tone or heart rate characterized by a sudden failure of the autonomic nervous system to maintain blood pressure and sometimes heart rate at a sufficient level to insure cerebral perfusion and consciousness. Conditions in this group comprise neurocardiogenic (vasovagal) syncope, situational syncope, carotid sinus syndrome and glossopharyngian neuralgia [3]. Malignancy-related reflex syncope is rare: in a series of 4,500 patients with cancer of the head and neck, the prevalence of syncope was estimated at $0.4 \%$ [4]. Patients with advanced tumoral disease at diagnosis and those with a history of neck surgery appear to be at greater risk of developing syncope [4]. Syncope may be an 
early sign of neck or cervical cancer [5] and even the sole sign of recurrent disease [6]. Head and neck cancer is the most frequent malignancy associated with syncope. However, cases of lymphomas [7], neurofibromas or metastatic breast [8] or lung [9] malignancies have been reported. To our knowledge, no case of metastatic esophagus cancer has been reported.

The pathophysiological mechanism involved in syncope is complex. The reflex arc of the carotid sinus is composed of an afferent pathway arising from baroreceptors located just above the bifurcation of the carotid artery. In case of arterial wall stretching, the information is transmitted by the carotid sinus nerve [a branch of the glossopharyngeal nerve (9th cranial nerve)] to brainstem centers in the caudal nucleus of the solitary tract. Interneurons then activate the efferent pathways resulting in the stimulation of the parasympathetic and the inhibition of the sympathetic systems leading to lower blood pressure and heart rate ( $\underline{\text { fig. }}$ ). Overstimulation of this reflex caused by mechanical traction or invasion of the carotid sinus by a tumor, or caused by tumor-induced irritation of the glossopharyngeal nerve (glossopharyngeal neuralgia) may produce symptoms. Malignant reflex syncope is often not reproducible by carotid sinus massage [4]. Patients with glossopharyngeal neuralgia usually have acute unilateral head or neck pain prior to syncopal attacks. The cardiovascular responses of neurally mediated syncope are classified into three types: cardioinhibitory response (asystole or symptomatic bradycardia associated with hypotension or not), vasodepressor response (hypotension without bradycardia), and a mixed response with features of both. Pure vasodepressor form, as evidenced in our patient by the upright tilt-table test, is thought to account for only $5-10 \%$ of cases of reflex syncope, although its prevalence increases up to $59 \%$ in the context of malignant forms [4].

Management is individual and depends on the type of syncope and symptoms. A large variety of medications have been used, including anticholinergics, vasoconstrictors, volume expanders, such as fludrocortisone, and antiepileptic drugs, such as carbamazepine, especially in case of glossopharyngeal neuralgia. Cardiac pacing may be ineffective in reflex syncope due to malignancy in which a mixed-response type (cardioinhibitory and vasodepressor) is usually present and may even promote emergence of vasodepressor manifestation [10]. Surgical treatment includes intracranial glossopharyngeal and vagal neurectomy [11] and is reserved for patients in whom standard treatment failed. Depending on the type of cancer, several cases of complete resolution of symptoms with chemotherapy have been reported [12, 13]. In our case, the patient could not be treated with chemotherapy, because the disease progressed despite multiple prior courses of chemotherapy. Radiation therapy may be effective by reducing the frequency of syncope, pain, and tumor size [14], but the procedure itself carries the risk of causing symptoms due to the application of pressure on the carotid sinus during the installation of the patient [15].

\section{Conclusion}

In conclusion, we reported the case of a patient with reflex vasodepressor syncope due to hypersensitivity carotid sinus secondary to recurrent, metastatic esophageal carcinoma, successfully relieved by radiotherapy. Because syncope may be an early sign of neck or cervical cancer and even the sole sign of recurrent disease, the diagnostic approach of 
syncope in patients with past history of cancer should include the possibility of neck tumor recurrence or metastasis and an oncologic workout should be considered. The patient presented in this report is a typical example of this condition.

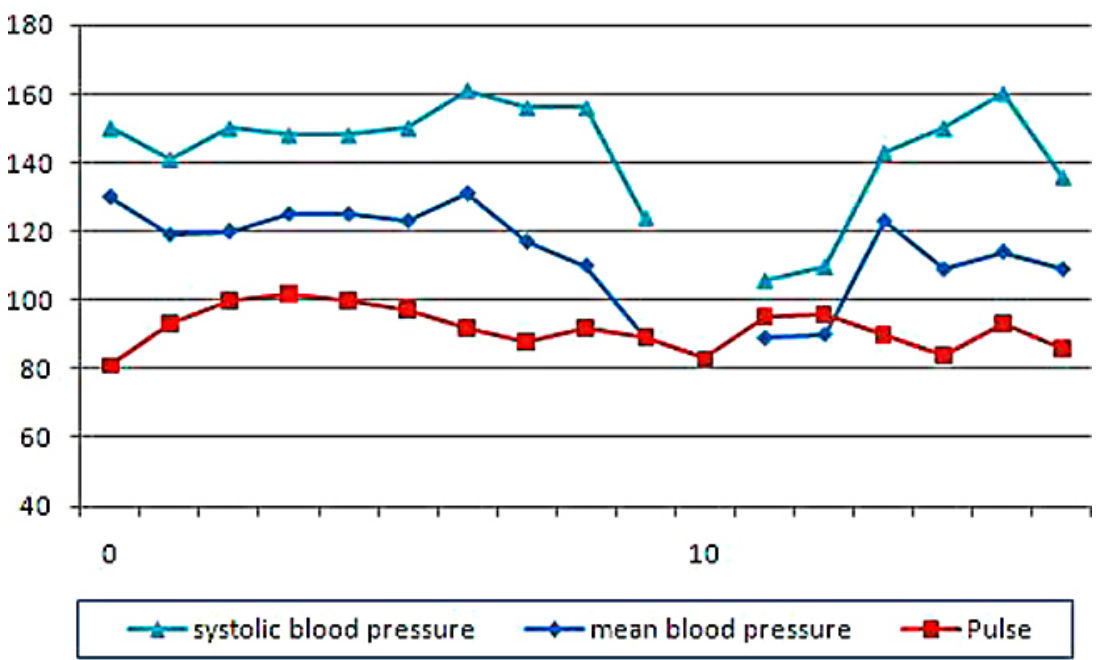

Fig. 1. Upright tilt-table test showing fall of blood pressure at $10 \mathrm{~min}$ with syncope.

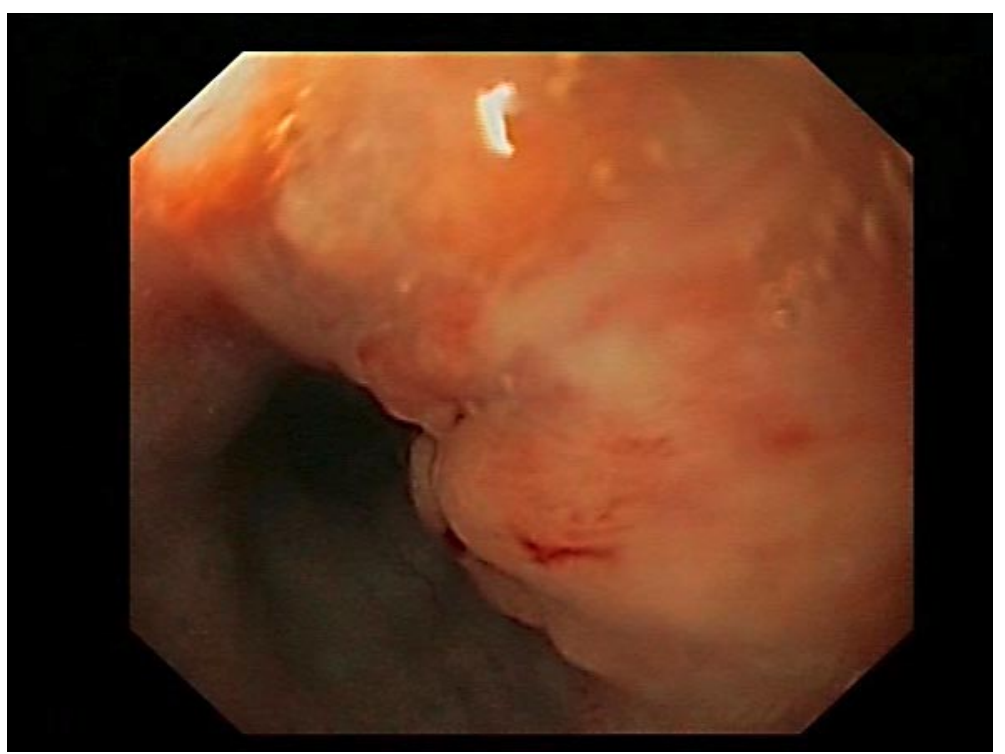

Fig. 2. Upper endoscopy showed tumor mass in the middle of esophagus. 


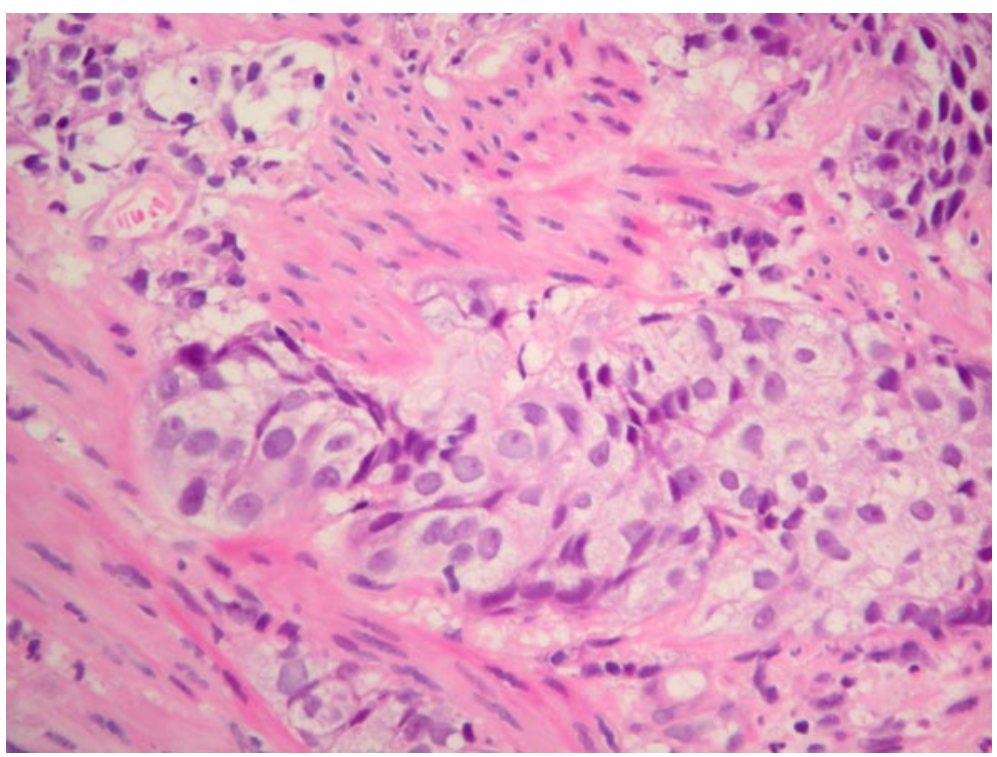

Fig. 3. Esophageal biopsy showed squamous cell carcinoma. H\&E, $\times 200$.

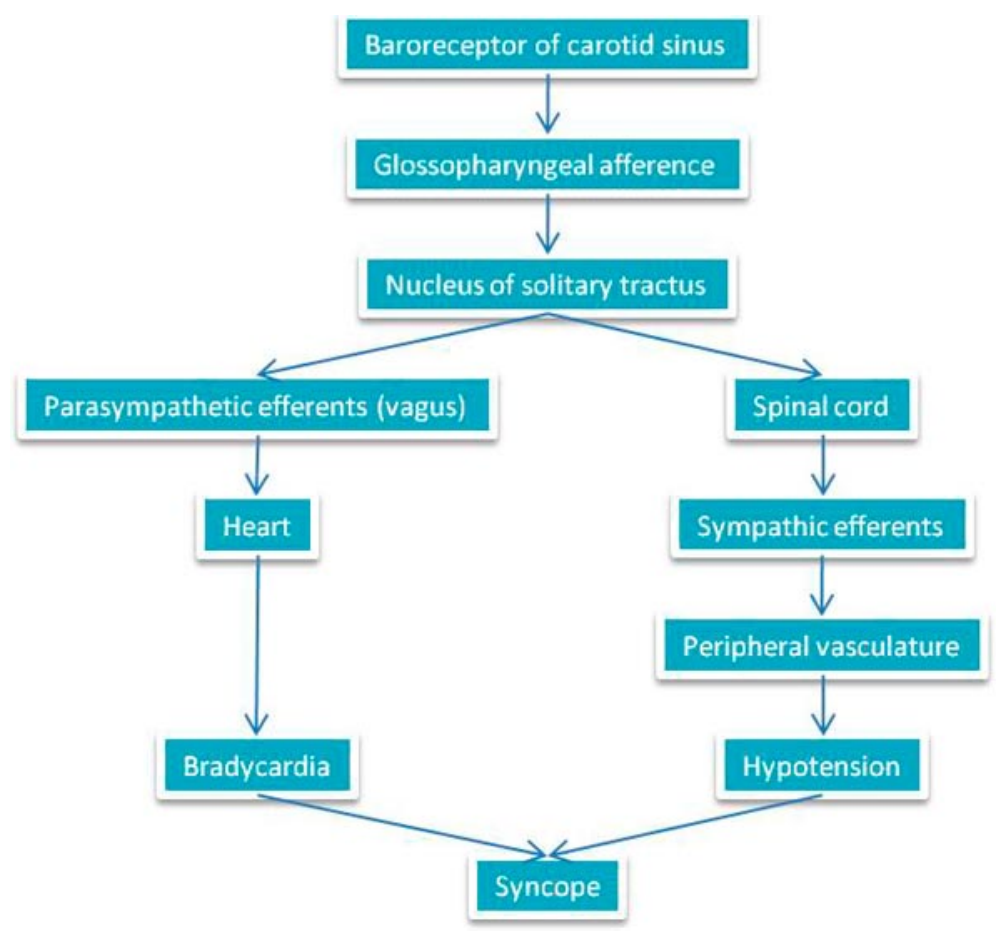

Fig. 4. Mechanism of syncope in malignant syncope. 


\section{References}

1 Sarasin FP, Louis-Simonet M, Carballo D, Slama S, Rajeswaran A, Metzger JT, Lovis C, Unger PF, Junod AF: Prospective evaluation of patients with syncope: a population-based study. Am J Med 2001;111:177-184.

-2 Moya A, Sutton R, Ammirati F, Blanc JJ, Brignole M, Dahm JB, Deharo JC, Gajek J, Gjesdal K, Krahn A, et al: Guidelines for the diagnosis and management of syncope (version 2009): the Task Force for the Diagnosis and Management of Syncope of the European Society of Cardiology (ESC). Eur Heart J 2009;30:2631-2671.

3 Kapoor WN: Syncope. N Engl J Med 2000;343:1856-1862.

4 Macdonald DR, Strong E, Nielsen S, Posner JB: Syncope from head and neck cancer. J Neurooncol 1983;1:257267.

5 Wang CH, Ng SH: Syncope as the initial presentation of nasopharyngeal carcinoma. J Neurooncol 1995;25:7375.

6 Ribeiro RT, Souza NA, Carvalho Dde S: Glossopharyngeal neuralgia with syncope as a sign of neck cancer recurrence. Arq Neuropsiquiatr 2007;65:1233-1236.

-7 Takahashi T, Obata N, Sugawara N, Fujita K, Oikawa K, Yoshimoto M, Urabe K, Nishimura M, Tsujisaki M: Carotid sinus syncope in a patient with relapsed cervical lymphoma. Int J Hematol 2006;84:92-93.

8 Holmes FA, Glass JP, Ewer MS, Terjanian T, Tetu B: Syncope and hypotension due to carcinoma of the breast metastatic to the carotid sinus. Am J Med 1987;82:1238-1242.

-9 Koga T, Kaseda S, Miyazaki N, Kawazoe N, Abe I, Sadoshima S, Onoyama K: Neurally mediated syncope induced by lung cancer - a case report. Angiology 2000;51:263-267.

10 Patel AK, Yap VU, Fields J, Thomsen JH: Carotid sinus syncope induced by malignant tumors in the neck. Emergence of vasodepressor manifestations following pacemaker therapy. Arch Intern Med 1979;139:12811284.

11 Lin RH, Teng MM, Wang SJ, Yeh TP, Liao KK, Liu HC: Syncope as the presenting symptom of nasopharyngeal carcinoma. Clin Neurol Neurosurg 1994;96:152-155.

12 Choi YM, Mafee MF, Feldman LE: Successful treatment of syncope in head and neck cancer with induction chemotherapy. J Clin Oncol 2006;24:5332-5333.

13 Chen-Scarabelli C, Kaza AR, Scarabelli T: Syncope due to nasopharyngeal carcinoma. Lancet Oncol 2005;6:347-349.

14 Tulchinsky M, Krasnow SH: Carotid sinus syndrome associated with an occult primary nasopharyngeal carcinoma. Arch Intern Med 1988;148:1217-1219.

15 Hong AM, Pressley L, Stevens GN: Carotid sinus syndrome secondary to head and neck malignancy: case report and literature review. Clin Oncol (R Coll Radiol) 2000;12:409-412. 